\title{
Improving Signal Quality in a Spectrum-Sliced WDM System Using SOA-Based Noise Reduction
}

\author{
Anoma D. McCoy, Peter Horak, Benn C. Thomsen, Morten Ibsen, M. R. Mokhtar, and D. J. Richardson
}

\begin{abstract}
We present an experimental and numerical study into the filtering effects in a spectrum-sliced incoherent light system incorporating a semiconductor optical amplifier (SOA) for noise reduction. Techniques to reduce the signal degradation caused by post-SOA filtering are investigated. The alignment of the receiver/demultiplexer filter relative to the input spectrum-slice is demonstrated to have a noticeable impact on the received signal quality. We also show that the linewidth enhancement factor $\alpha$ of the SOA has a marked effect on the observed spectral distortion, which results in a strong dependence of the received signal quality on $\alpha$. Our results illustrate the potential to improve system performance by optimized SOA design.
\end{abstract}

Index Terms-Optical noise, semiconductor optical amplifier (SOA), spectrum slicing, wavelength-division multiplexing (WDM).

\section{INTRODUCTION}

$\mathbf{T}$ HE GROWING demand for high bandwidth access services is driving the development of low-cost passive optical network technology such as spectrum-sliced wavelength-division multiplexing (WDM) using incoherent sources [1]. However, the intensity noise present in incoherent light imposes an upper limit on the achievable system performance. This impacts high channel density spectrum-sliced systems, as the intensity noise increases with decreasing channel bandwidth. A gain-saturated semiconductor optical amplifier (SOA) can be used to overcome this limitation and suppress the intensity noise, allowing for increased spectral efficiency [2]. This technique has the additional benefit that the SOA can be used simultaneously for both signal modulation and amplification.

However, filtering after the SOA (e.g., in a WDM demultiplexer) reduces the noise suppression offered by the SOA, degrading the received signal quality [3]. This effect is more pronounced for narrow channel widths, where the nonlinearities of the SOA cause significant spectral distortion and broadening. This limits the achievable channel granularity due to the tradeoff between intensity noise and crosstalk [4].

In this letter, we study the effects of post-SOA filtering, using numerical simulations to support our experimental observations. We discuss techniques to improve the received signal quality, enhancing the potential of SOA-based noise reduction in high channel density spectrum-sliced systems.

Manuscript received June 10, 2004; revised August 16, 2004.

The authors are with the Optoelectronics Research Centre, University of Southampton, Southampton SO17 1BJ, U.K. (e-mail: adm@orc.soton.ac.uk).

Digital Object Identifier 10.1109/LPT.2004.837916

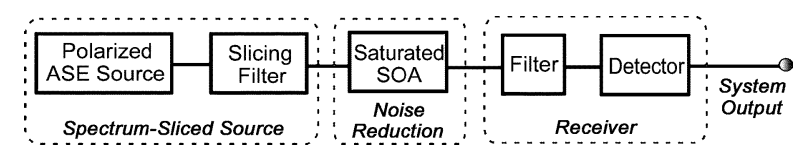

Fig. 1. Single-channel system block diagram.

\section{NUMERICAL SimULATION}

To understand the effects of post-SOA filtering on the intensity-smoothed light, the system shown in Fig. 1 was modeled using the field $E(z, t)$ and carrier density $N(z, t)$ equations [5]

$$
\begin{aligned}
\frac{\partial E}{\partial z}+\frac{1}{v_{g}} \frac{\partial E}{\partial t} & =\frac{\Gamma}{2}(1-i \alpha) g(N) E-\frac{\alpha_{\text {int }}}{2} E \\
\frac{\partial N}{\partial t} & =\frac{I}{q V}-\frac{N}{\tau}-\frac{\Gamma}{\hbar \omega_{o}} g(N)|E|^{2}
\end{aligned}
$$

where $v_{g}$ is the group velocity, $\Gamma$ the mode confinement, $\alpha$ the linewidth enhancement factor, $g(N)$ the gain coefficient, $\alpha_{\text {int }}$ the internal loss, $I$ the injected current, $q$ the electronic charge, $V$ the active layer volume of the device, $\tau$ the spontaneous carrier lifetime, and $\omega_{o}$ the input center frequency. Although the noise reduction can be modeled by the intensity and carrier density rate equations alone [6], the field equation is required to predict the spectral properties of the SOA output [7], including the effects of post-SOA filtering.

For our traveling-wave SOA model, the spectrum-sliced input field is modeled using thermal light statistics. The amplifier is divided into short segments where the length of each segment is small relative to the spatial variations of the input intensity. The rate equations are solved on this spatial grid using an ordinary differential equation solver which calculates the field at the device output, $E(t, z=L)$. The model neglects the amplified spontaneous emission (ASE) noise added by the SOA.

The equations above consider only the interband effects of carrier density pulsations and ignore carrier heating and spectral hole burning. However, the good agreement between experiment and simulations (presented in Section III) suggests that our model effectively represents the nonlinear interactions producing the noise suppression and subsequent filtering effects.

\section{RESUlTS AND DISCUSSION}

We first consider the spectral broadening effects introduced by the SOA for varying spectrum-sliced input bandwidths. For all characterizations presented here, the SOA was operated at a drive current of $200 \mathrm{~mA}$ and a constant input power of $+5 \mathrm{dBm}$ to ensure deep saturation. As seen in Fig. 2, the numerical model accurately predicts the output spectra, clearly showing 

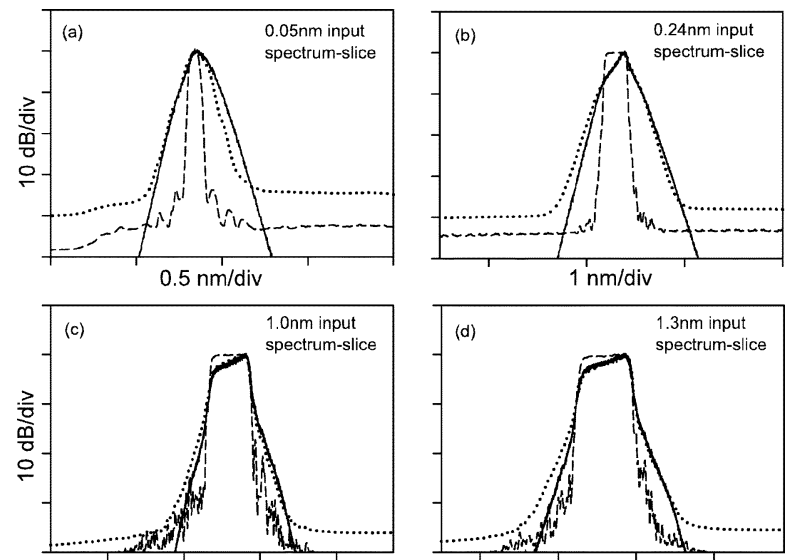

$2 \mathrm{~nm} / \mathrm{div}$

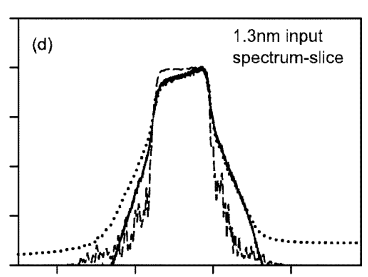

$2 \mathrm{~nm} / \mathrm{div}$

--- SOA Input $\quad$-..... SOA output (expt) - SOA output (sim)

Fig. 2. Spectra before and after the saturated SOA for 0.05-, 0.24-, 1.0-, and $1.3-\mathrm{nm}$ (3-dB bandwidth) filters. Input slices are centered at approximately $1551 \mathrm{~nm}$.

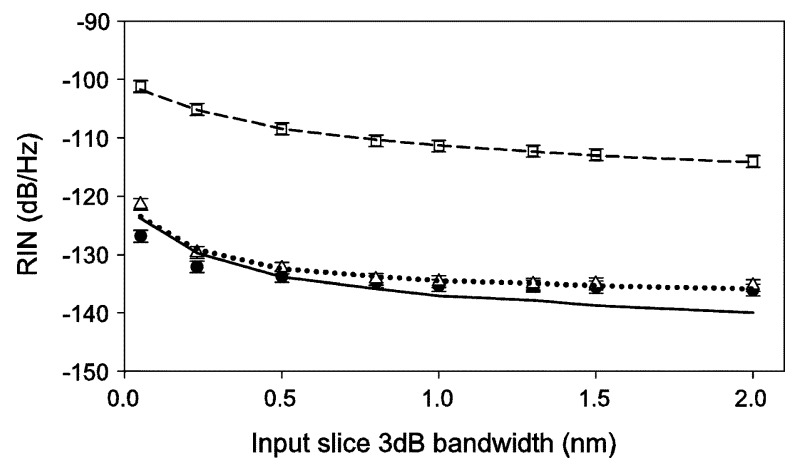

Fig. 3. Measured $(\square)$ and predicted RIN $(--)$ at SOA input, measured (•) and predicted RIN (-) at SOA output, measured SOA output RIN after ASE filter $(\Delta)$, and predicted RIN at SOA output with detector floor $(.$.$) as a$ function of input filter bandwidth.

the broadening and distortion observed in the experimental measurements. The main discrepancy is the ASE floor, which is neglected in the simulations. The spectral broadening at the SOA output is caused by phase modulation-induced chirp [5] and four-wave mixing (FWM). The relative efficiencies of the two processes depend on the input slice bandwidth; wider slices generate more FWM products, while the chirp is more pronounced in the narrower spectral slices where the larger intensity fluctuations produce greater phase noise [7].

Relative intensity noise (RIN) measurements before and after the SOA for the input spectrum-slices shown above are given in Fig. 3, along with results for additional slice widths of 0.5 , $0.8,1.5$, and $2.0 \mathrm{~nm}$ (all filter transfer functions are similar in shape). The RIN measurements were performed at $100 \mathrm{MHz}$, using a 125-MHz high sensitivity photodetector. The numerical model gives good agreement with measured values. For bandwidths above $0.8 \mathrm{~nm}$, the discrepancies are due to the detector noise floor in the measurement; once the detector floor is factored into the simulation, the predicted values closely match the experimental results. For narrower bandwidths where the measurements are not detector noise limited, the SOA output RIN is lower than simulations. However, removing ASE using a 5-nm filter increases the RIN, giving better agreement with the model

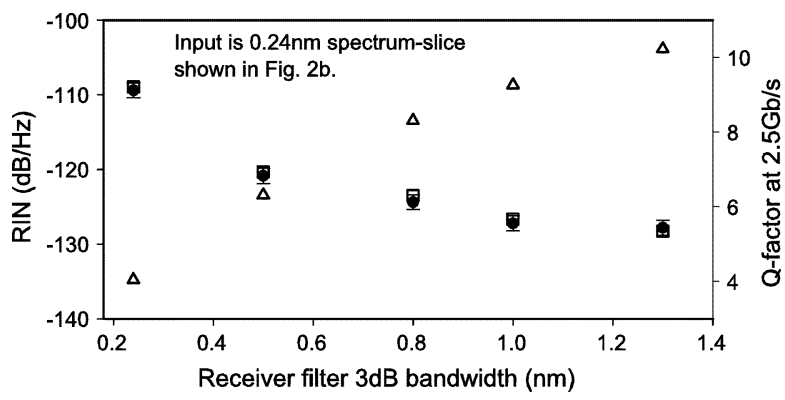

Fig. 4. Measured (•) and predicted ( $\square$ ) RIN at system output for varying receiver filters (receiver filter aligned to SOA input spectrum-slice). Filter transfer functions are as shown in Fig. 2. Simulation results include detector floor. System $Q(\Delta)$ at a received power of $0 \mathrm{dBm}$ is shown for comparison.

which does not include amplifier ASE. This suggests that the added ASE in fact aids in the noise suppression process. Our results also show that the SOA output RIN improves with increasing input slice bandwidth. This is in contrast with previous results [2] where the SOA output signal-to-noise ratio was observed to be independent of input bandwidth.

We also characterized the RIN of the post-SOA filtered signal as a function of receiver filter bandwidth (Fig. 4), and obtain excellent agreement between experimental and simulation results. The observed noise increase is caused by spectral filtering at the receiver. The nonlinearities that occur within the gain-saturated SOA produce interactions between the various spectral components present in the amplifier (this includes new frequencies generated by the nonlinear processes, added ASE, and the original input signal) [8]. These nonlinear interactions introduce correlation between the spectral components in the amplifier output light, which results in noise suppression. Filtering this signal reduces the correlation, and thus, increases the noise level. $Q$ measurements at $2.5 \mathrm{~Gb} / \mathrm{s}$ are also included in Fig. 4 to give a qualitative indication of the system performance degradation due to post-SOA filtering. The signal RIN must be less than approximately $-125 \mathrm{~dB} / \mathrm{Hz}$ to obtain a $Q \geq 8$ (bit-error rate $\leq 10^{-15}$ ). This requires the receiver bandwidth to be greater than three times the input slice bandwidth, thus limiting the channel density and spectral efficiency.

In view of these results, we now discuss techniques to improve signal quality in a spectrum-sliced system incorporating SOA-based noise reduction.

As observed in Fig. 2, a shift in the peak wavelength occurs as the light travels through the saturated amplifier. This red-shift is the result of phase modulation in the SOA [5]. By positioning the post-SOA receiver filter to account for this frequency shift relative to the input spectrum-slice, we observed a noticeable improvement in signal quality. Fig. 5 shows the RIN at the system output as a function of the frequency offset between the 0.24-nm input spectrum-slice and the receiver filter. The lowest RIN is obtained when the receiver filter is aligned $\sim 10 \mathrm{GHz}(0.08 \mathrm{~nm})$ below the center frequency of the input spectrum-slice. This illustrates the importance of aligning the receiver filter to the SOA output in narrow-channel systems.

As the spectral distortion introduced by the saturated amplifier is largely due to the device linewidth enhancement factor $\alpha$, we next used the model to assess the impact of $\alpha$ on the received signal quality. Using a $0.24-\mathrm{nm}(3 \mathrm{~dB})$ input spectrum slice, we 


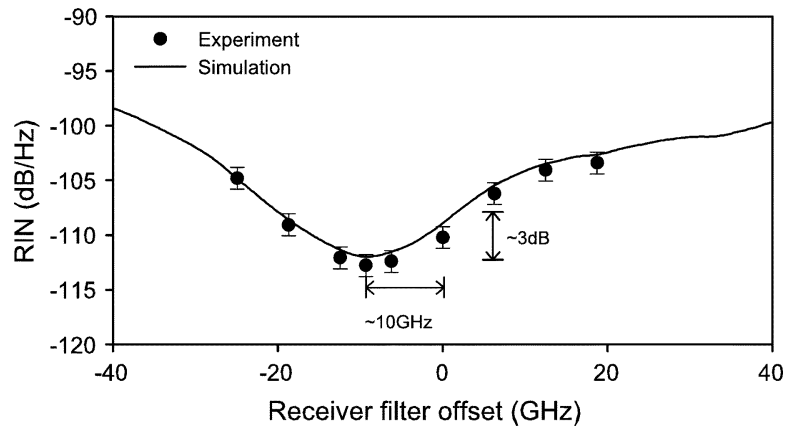

Fig. 5. RIN at receiver filter output, as a function of frequency offset between 0.24 -nm input spectrum-slice and 0.24-nm receiver filter (same shape as slicing filter).

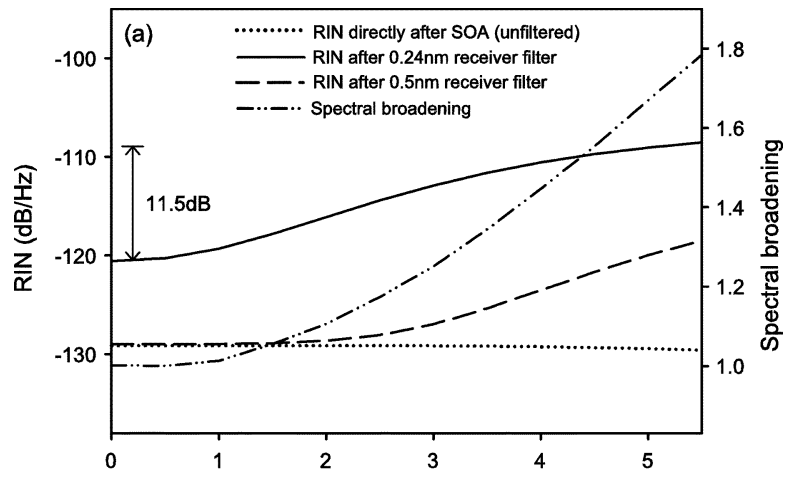

Linewidth enhancement factor, $\alpha$

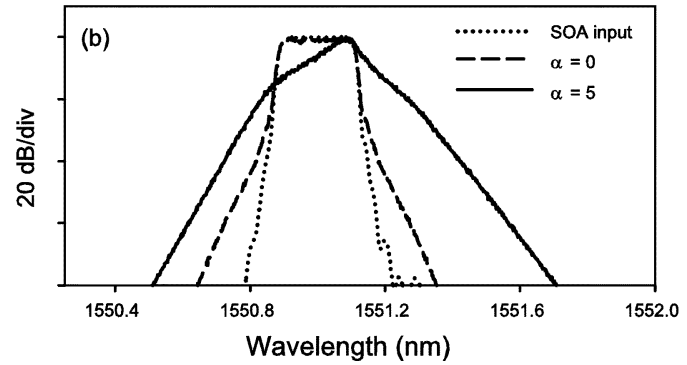

Fig. 6. Simulation results for varying $\alpha$. (a) Unfiltered SOA output RIN, RIN at filter output (0.24- and 0.5 -nm filters), and spectral broadening (ratio of SOA output $10-\mathrm{dB}$ bandwidth to input 10-dB bandwidth). (b) SOA input spectrum and output spectra for $\alpha=0$ and 5 .

calculate the RIN as a function of $\alpha$ directly after the SOA and at the output of the receiver filter. Simulation results are shown in Fig. 6(a), for post-SOA receiver filters (aligned to input slice) of 0.24- and 0.5-nm ( $3 \mathrm{~dB})$ bandwidths.

As expected [7], the noise suppression directly after the SOA [shown by the dotted curve in Fig. 6(a)] is not affected by $\alpha$. However, $\alpha$ clearly has a strong effect on the post-SOA filtered signal quality. Typical bulk SOAs have linewidth enhancement factors between 3 and 8 ( $\alpha=5$ was used as a best fit to our experimental data). Various techniques have been investigated to reduce the linewidth enhancement factor in semiconductor laser diodes [9]-[11]; optimized device structures have been shown capable of yielding linewidth enhancement factors less than 1 . By reducing $\alpha$ in our simulations, we obtain a maximum RIN improvement of $11.5 \mathrm{~dB}$ over our experimental values for the $0.24-\mathrm{nm}$ filter and a 9-dB improvement with the $0.5-\mathrm{nm}$ filter.
The spectral broadening at the SOA output is also shown relative to the input slice bandwidth, illustrating the significant influence of the SOA linewidth enhancement factor on spectral efficiency. As $\alpha$ is reduced, the spectral broadening due to the phase modulation-induced chirp decreases as does the FWM [Fig. 6(b)]. This reduces the impact of post-SOA filtering, resulting in improved RIN. The broadening observed in the tails of the spectrum for $\alpha=0$ is due to remaining FWM contributions. Our results clearly show that the post-SOA filtered signal quality can be improved significantly by reducing the linewidth enhancement factor.

\section{CONCLUSION}

We have investigated techniques to improve the received signal quality in spectrum-sliced systems incorporating SOA-based noise reduction. Spectral distortion and post-SOA filtering effects have been examined experimentally and numerically. Aligning the receiver filter to compensate for the red-shift introduced by the saturated SOA produced an improvement of $\sim 3 \mathrm{~dB}$ in the signal RIN. Using simulations, we have also demonstrated that reducing the linewidth enhancement factor of the amplifier can improve the received signal quality by up to $11.5 \mathrm{~dB}$. These optimizations will increase system performance and allow for greater channel granularity and spectral-efficiency in high channel count spectrum-sliced systems using gain-saturated SOAs.

\section{REFERENCES}

[1] K. Akimoto, J. Kani, M. Teshima, and K. Iwatsuki, "Super-dense WDM transmission of spectrum-sliced incoherent light for wide-area access network," J. Lightw. Technol., vol. 21, no. 11, pp. 2715-2721, Nov. 2003.

[2] S.-J. Kim, J.-H. Han, J.-S. Lee, and C.-S. Park, "Intensity noise suppression in spectrum-sliced incoherent light communication systems using a gain saturated semiconductor optical amplifier," IEEE Photon. Technol. Lett., vol. 11, no. 8, pp. 1042-1044, Aug. 1999.

[3] A. D. McCoy, B. Thomsen, M. Ibsen, and D. J. Richardson, "Experimental study on receiver filtering effects in a spectrum-sliced incoherent light WDM system using SOA based noise reduction," in Proc. Eur. Conf. Opt. Commun. (ECOC 2003), Paper We4P155, pp. 866-867.

[4] A. D. McCoy et al., "Filtering effects in a spectrum-sliced WDM system using SOA-based noise reduction," IEEE Photon. Technol. Lett., vol. 16, no. 2, pp. 680-682, Feb. 2004.

[5] G. P. Agrawal and N. A. Olsson, "Self-phase modulation and spectral broadening of optical pulses in semiconductor laser amplifiers," IEEE J. Quantum Electron., vol. 25, no. 11, pp. 2297-2306, Nov. 1989.

[6] M. Zhao, G. Morthier, and R. Baets, "Analysis and optimization of intensity noise reduction in spectrum-sliced WDM systems using a saturated semiconductor optical amplifier," IEEE Photon. Technol. Lett., vol. 14, no. 3, pp. 390-392, Mar. 2002.

[7] M. Munroe et al., "Spectral broadening of stochastic light intensity-smoothed by a saturated semiconductor optical amplifier," IEEE J. Quantum Electron., vol. 34, no. 3, pp. 548-551, Mar. 1998.

[8] M. Shtaif and G. Eisenstein, "Experimental study of the statistical properties of nonlinearly amplified signals in semiconductor optical amplifiers," IEEE Photon. Technol. Lett., vol. 9, no. 7, pp. 904-906, Jul. 1997.

[9] M. P. Mullane and J. McInerney, "Sensitivity of the linewidth enhancement factor to structure and threshold level in strained semiconductor lasers," in Proc. Conf. Lasers and Electro-Optics (CLEO 2000), Paper CTuA60, pp. 180-181.

[10] T. Yamanaka et al., "Theoretical study on enhanced differential gain and extremely reduced linewidth enhancement factor in quantum-well lasers," IEEE J. Quantum Electron., vol. 29, no. 6, pp. 1609-1616, Jun. 1993.

[11] P. K. Kondratko et al., "Observations of near-zero linewidth enhancement factor in a quantum-well coupled quantum-dot laser," Appl. Phys. Lett., vol. 83, pp. 4818-4820, 2003. 